\title{
trans-Anethole of Fennel Oil is a Selective and Nonelectrophilic Agonist of the TRPA1 Ion Channel\$
}

\author{
DTosifa Memon, Oleg Yarishkin, Christopher A. Reilly, David Križaj, Baldomero M. Olivera, \\ and Russell W. Teichert
}

\author{
Departments of Biology (T.M., B.M.O., R.W.T.), Pharmacology and Toxicology (T.M., C.A.R.), and Ophthalmology and Visual \\ Sciences (O.Y., D.K.), University of Utah, Salt Lake City, Utah
}

Received September 16, 2018; accepted January 18, 2019

\begin{abstract}
Transient receptor potential (TRP) cation channels are molecular targets of various natural products. TRPA1, a member of TRP channel family, is specifically activated by natural products such as allyl isothiocyanate (mustard oil), cinnamaldehyde (cinnamon), and allicin (garlic). In this study, we demonstrated that TRPA 1 is also a target of trans-anethole in fennel oil (FO) and fennel seed extract. Similar to FO, trans-anethole selectively elicited calcium influx in TRPA1-expressing mouse sensory neurons of the dorsal root and trigeminal ganglia. These FOand anethole-induced calcium responses were blocked by a selective TRPA1 channel antagonist, HC-030031. Moreover, both FO and trans-anethole induced calcium influx and
\end{abstract}

transmembrane currents in HEK293 cells stably overexpressing human TRPA1 channels, but not in regular HEK293 cells. Mutation of the amino acids S873 and T874 binding site of human TRPA1 significantly attenuated channel activation by trans-anethole, whereas pretreating with glutathione, a nucleophile, did not. Conversely, activation of TRPA1 by the electrophile allyl isothiocyanate was abolished by glutathione, but was ostensibly unaffected by mutation of the ST binding site. Finally, it was found that trans-anethole was capable of desensitizing TRPA1, and unlike allyl isothiocyanate, it failed to induce nocifensive behaviors in mice. We conclude that trans-anethole is a selective, nonelectrophilic, and seemingly less-irritating agonist of TRPA1.

\section{Introduction}

The oil of fennel, or fennel oil (FO), is extracted from seeds of Foeniculum vulgare, a flavorful herb used in cooking, baking, herbal teas, and alcoholic beverages (Badgujar et al., 2014). Fennel oil consists of multiple chemical components, of which trans-anethole (referred to as simply anethole for the remainder of this article) has been reported to account for $\sim 70 \%-80 \%$ of the oil (Badgujar et al., 2014). Anethole, an aromatic compound, is a major component not only of fennel

This work was supported by the National Institutes of Health National Institute of General Medical Sciences [Grant GM48677], National Institute of Environmental Health Sciences [Grant ES017431], and National Eye Institute [Grants EY022076, EY027920, and EY014800]. This work was also supported by the Assistant Secretary of Defense for Health Affairs, through the Peer Reviewed Medical Research Program [Award W81XWH-17-1-0413], and by unrestricted support from Research to Prevent Blindness to the Moran Eye Institute at the University of Utah. Opinions, interpretations, conclusions, and recommendations are those of the author and are not necessarily endorsed by the Department of Defense. The US Army Medical Research Acquisition Activity, 820 Chandler Street, Fort Detrick MD 21702-5014, is the awarding and administering acquisition office. In conducting research using animals, the investigator(s) adhered to the laws of the United States and regulations of the Department of Agriculture. The authors declare no competing financial interests.

https://doi.org/10.1124/mol.118.114561.

S This article has supplemental material available at molpharm. aspetjournals.org. oil but also of star anise oil (Ritter et al., 2013). Anethole has been shown to have antibacterial and antifungal properties in vitro, as well as anti-inflammatory and analgesic activity in vivo (Ritter et al., 2013; Badgujar et al., 2014). The beneficial properties of anethole and FO together with the pleasant flavor might account for its use for culinary purposes across cultures and in treating diseases (Badgujar et al., 2014). However, the molecular mechanisms underlying the desirable and medicinal properties of anethole remain unknown.

Over the past two decades, transient receptor potential (TRP) channels have emerged as sensors and mediators of cellular responses to numerous odorants and tastants, such as menthol (TRPM8 and A1), mustard oil [aka, allyl isothiocyanate (AITC); TRPA1], carvacrol (TRPA1 and V3), and capsaicin (TRPV1) (Caterina et al., 1997; Jordt et al., 2004; Bautista et al., 2007; Roper, 2014; Friedland and Harteneck, 2017). Hence, we hypothesized that the TRP channel(s) may also be a molecular target of FO, and specifically anethole. This study demonstrated that anethole is a selective agonist of mouse and human TRPA1. Further, our results identify TRPA1 as the principal mediator of FO- and anethole-elicited responses in somatosensory neurons via interactions with the previously characterized menthol/propofol binding site involving amino acids S873 and T874 (Xiao et al., 2008). Finally, despite robust

ABBREVIATIONS: AITC, allyl isothiocyanate; CGRP, calcitonin gene-related peptide; DRG, dorsal root ganglia; DRG Obs., DRG observation solution; FO, fennel oil; GC-MS, gas chromatography-mass spectrometry; GFP, green fluorescent protein; GSH, glutathione; hTRPA1, human TRPA1; IB4, isolectin B4; TG, trigeminal ganglia; ST, amino acids S873 and T874; TRITC, tetramethylrhodamine isothiocyanate; TRP, transient receptor potential; TRPA1, transient receptor potential cation channel subfamily A member 1; TRPM8, transient receptor potential melastatin-8; TRPV1, transient receptor potential vanilloid-1; TRPV3, transient receptor potential vanilloid-3. 
activation of TRPA1, anethole appears to be non- or lessirritating than AITC.

\section{Materials and Methods}

Cell Culture. Dorsal root ganglia (DRG), trigeminal ganglia (TG), and HEK293 cell cultures were prepared as described previously (Deering-Rice et al., 2011; Teichert et al., 2012a, 2014; Memon et al., 2017). In brief, lumbar DRG and TG were removed from adult ( $\geq 45$ days old) wild-type C57BL/6 or calcitonin gene-related peptide (CGRP)-green fluorescent protein (GFP) mice on a CD-1 genetic background (Gong et al., 2003), trypsinized with $0.25 \%$ trypsin, and plated in 24-well poly-D-lysine-coated plates. Cultured neurons of DRG and TG were incubated overnight at $37^{\circ} \mathrm{C}$ with minimum essential medium supplemented with $10 \%$ fetal bovine serum, $1 \times$ penicillin/streptomycin, $10 \mathrm{mM}$ HEPES, and $0.4 \%$ (w/v) glucose, $\mathrm{pH}$ 7.4. Similarly, HEK293 cells were plated in poly-D-lysine-coated plates and incubated overnight at $37^{\circ} \mathrm{C}$ with Dulbecco's modified Eagle's medium:F12 medium supplemented with $5 \%$ fetal bovine serum and $1 \times$ penicillin/streptomycin. HEK293 cells overexpressing human TRPA1 or GCaMP6 were cultured similarly with the addition of $300 \mu \mathrm{g} / \mathrm{ml}$ Geneticin to the medium. For experiments, HEK293 cells were plated in a 96 -well plate coated with $1 \%(w / v)$ gelatin. HEK293 cells overexpressing GCaMP6 (HEK-GCaMP6) were used for transient transfection studies of human TRPA1 mutants using Lipofectamine 2000 (Invitrogen) as described previously (Deering-Rice et al., 2011, 2015; Shapiro et al., 2013).

Calcium Imaging. Prior to calcium imaging experiments, neurons and HEK293 cells were incubated with Fura 2-AM for 1 hour at $37^{\circ} \mathrm{C}$ and then 30 minutes at room temperature. Changes in intracellular calcium content were monitored as a ratio of emission at $510 \mathrm{~nm}$ when excited alternately by 340 and $380 \mathrm{~nm}$ of light [340/380 nm ratio ( $y$-axis)] over time in minutes ( $x$-axis) as described previously (Teichert et al., 2014; Memon et al., 2017). A physiologic salt solution termed "DRG observation solution" (DRG Obs.) was composed of $145 \mathrm{mM} \mathrm{NaCl}, 5 \mathrm{mM} \mathrm{KCl}, 2 \mathrm{mM} \mathrm{CaCl}, 1 \mathrm{mM} \mathrm{MgCl}$, $1 \mathrm{mM}$ sodium citrate, $10 \mathrm{mM}$ HEPES, and $10 \mathrm{mM}$ glucose, $\mathrm{pH} 7.4$, and was used as the bath solution and to dissolve chemicals at working concentrations (Memon et al., 2017). Fennel oil (Plant Therapy) and trans-anethole (Sigma-Aldrich) solutions were made fresh in DRG Obs. containing $0.1 \mathrm{mg} / \mathrm{ml}$ bovine serum albumin before every experiment. All of the treatments including the depolarizing pulse of $30 \mathrm{mM}$ extracellular potassium $\left(\left[\mathrm{K}^{+}\right]_{\mathrm{O}}\right)$, fennel oil, anethole, menthol, AITC, and capsaicin were applied to neurons or HEK293 cells for 15 seconds, with the exception of the TRPA1 antagonist HC-030031, which was preincubated for 7 minutes at room temperature prior to the application of various stimuli.

To identify CGRP-expressing peptidergic neurons, DRG neurons cultured from CGRP-GFP transgenic CD-1 mice were imaged with a GFP filter set prior to calcium imaging experiments. To identify nonpeptidergic neurons, neurons were incubated with Alexa Fluor 568 isolectin GS-IB4 conjugate (Invitrogen) for 5 minutes at room temperature followed by three washes. Isolectin B4 (IB4)-stained neurons were then imaged with a TRITC (tetramethylrhodamine isothiocyanate) filter set.

Calcium imaging of transiently transfected HEK-GCaMP6overexpressing cells was also performed as described previously (Deering-Rice et al., 2011, 2012; Lamb et al., 2017). In brief, HEKGCaMP6 cells were transfected prior to studies as described earlier. Changes in GCaMP6 fluorescence due to calcium binding within cells was measured over time microscopically using an EVOS-FL (Life Technologies) auto and a GFP filter cube. Treatments of $100 \mu \mathrm{M}$ AITC, $1.3 \mathrm{mM}$ anethole, $20 \mathrm{mM}$ glutathione (GSH), and $10 \mu \mathrm{M}$ ionomycin were prepared in LHC-9 cell culture medium containing $0.1 \mathrm{mg} / \mathrm{ml}$ bovine serum albumin and added to cells maintained at $37^{\circ} \mathrm{C}$ in a $95 \%$ air $+5 \% \mathrm{CO}_{2}$ atmosphere. For experiments using GSH, AITC and anethole were incubated with $20 \mathrm{mM}$ GSH for 10 minutes before application.
Electrophysiology. Patch-clamp data were recorded with a Multiclamp 700B amplifier (Molecular Devices) using a Digidata 1550 digitizer and Clampex 10.5 acquisition software (Molecular Devices), as reported previously (Jo et al., 2016; Phuong et al., 2017). Data were acquired at $5 \mathrm{kHz}$ and filtered at $2 \mathrm{kHz}$ with an 8-pole Bessel filter. Results were analyzed with Clampfit 10.5 software (Molecular Devices) and OriginPro 8 (Origin Laboratory). Patch pipettes were pulled from borosilicate glass capillaries (WPI) using a P-2000 laser pooler (Sutter Instruments). If not specified, the holding potential was $-40 \mathrm{mV}$. Ramps from -100 to $100 \mathrm{mV}$ (1-second duration) were applied every 5 seconds. $\mathrm{EC}_{50}$ was calculated by fitting experimental data points with logistic function:

$$
\mathrm{y}=\frac{\mathrm{A}_{1}-\mathrm{A}_{2}}{1+\left(\mathrm{x} / \mathrm{x}_{0}\right)^{\mathrm{p}}}+\mathrm{A}_{2}
$$

where $A_{1}$ stands for initial value, $A_{2}$ is the final value, $x_{0}$ is the center (or $\mathrm{EC}_{50}$ ), and $\mathrm{p}$ is power (slope factor).

The extracellular solution contained the following: $140 \mathrm{mM} \mathrm{NaCl}$, $2.5 \mathrm{mM} \mathrm{KCl}, 1.5 \mathrm{mM} \mathrm{MgCl}_{2}, 2.0 \mathrm{mM} \mathrm{CaCl}_{2}, 10 \mathrm{mM}$ HEPES, $10 \mathrm{mM}$ Dglucose, and $5 \mathrm{mM}$ tetraethylammonium chloride $(\mathrm{pH}=7.44$ adjusted with $\mathrm{NaOH}$ ). Osmolarity was 296-300 mOsm. The intracellular solution (pipette solution) contained the following: $135 \mathrm{mM}$ K-gluconate, $10 \mathrm{mM} \mathrm{KCl}, 1 \mathrm{mM} \mathrm{MgCl} 2,0.5 \mathrm{mM}$ EGTA, and $0.0001 \mathrm{mM} \mathrm{CaCl} \mathrm{m}_{2}$ ( $\mathrm{pH}=7.2$ was adjusted with $\mathrm{KOH})$. The osmolarity of 296-300 mOsm was adjusted with mannitol.

Gas Chromatography-Mass Spectrometry (CG-MS). GC-MS analysis of fennel oil and anethole was carried out by the University of Utah Metabolomics core facility. Samples $(1 \mu \mathrm{l})$ were resuspended into $1 \mathrm{ml}$ of EtOAc and transferred into a glass GC-MS autosampler vial for analysis. GC-MS analysis was conducted using an HP6890 instrument interfaced with an MSD-HP5973 detector and equipped with a Zebron ZB-5MSi Guardian column (30-m $\times$ 0.25-mm i.d., 0.25$\mu \mathrm{m}$ film thickness; Phenomenex) and an HP7682 injector. Helium was used as a carrier gas at a flow rate of $13.8 \mathrm{ml} / \mathrm{min}$ with a $10: 1$ split ratio at an injection volume of $1 \mu \mathrm{l}$. The injector temperature was $250^{\circ} \mathrm{C}$. The oven temperature gradient was programmed as follows: $50^{\circ} \mathrm{C}$ 1 minute, an increase in temperature at a rate of $40^{\circ} \mathrm{C} / \mathrm{min}$, held at $65^{\circ} \mathrm{C}$ for 1 minute, an increase in temperature at a rate of $40^{\circ} \mathrm{C} / \mathrm{min}$ to $330^{\circ} \mathrm{C}$, and held for 5 minutes. MS spectra were obtained in electron ionization mode from a range of $m / z 40-400$. The MS quad temperature was $150^{\circ} \mathrm{C}$, the $\mathrm{MS}$ source temperature was $230^{\circ} \mathrm{C}$, and a solvent cut time of 2.5 minutes was used. Data were collected at four scans/s. Data were first collected on an Agilent MSD Chemstation, translated using an Agilent GC MSD translator, then analyzed using Agilent MassHunter Quant and the NIST14 library.

Behavioral Assays. Adult wild-type C57BL/6 mice were subjected to subcutaneous injection of $20 \mu \mathrm{l}$ of vehicle (DRG Obs.), anethole $(6.6$ or $66 \mathrm{mM})$, or AITC $(10 \mathrm{mM})$ in the left hind paw. These mice were then monitored for 15 minutes post injection. Mice injected with AITC exhibit a pain response by licking or lifting the injected paw (Kwan et al., 2006; Everaerts et al., 2011). Thus, we recorded the duration and frequency of these nocifensive behaviors over a 15-minute observation period.

Data Analysis. Calcium imaging data were subjected to binary scoring as described in detail previously (Memon et al., 2017). A response to a given stimulus was considered positive if the $340 / 380 \mathrm{~nm}$ ratio change was $>0.05$ from baseline. The percentage of responsive neurons $\left(\left[\mathrm{K}^{+}\right]_{\mathrm{O}}\right.$ responsive) or HEK293 cells, averaged across multiple experiments from $\geq 3$ cultures, is presented as response frequency $\pm \mathrm{S}$. E.M. for a given stimulus (Figs. 1 and 2). The total number of sensory neurons or HEK293 cells scored for each experimental condition is indicated in the respective figure legends.

Anethole + , AITC + , and capsaicin + neurons, once scored, were then manually analyzed for GFP expression (in CGRP-expressing peptidergic neurons) and IB4 staining (nonpeptidergic neurons). For HEK-GCaMP6 cells, responses to agonist treatments were normalized to ionomycin response for each experiment and averaged across 
A
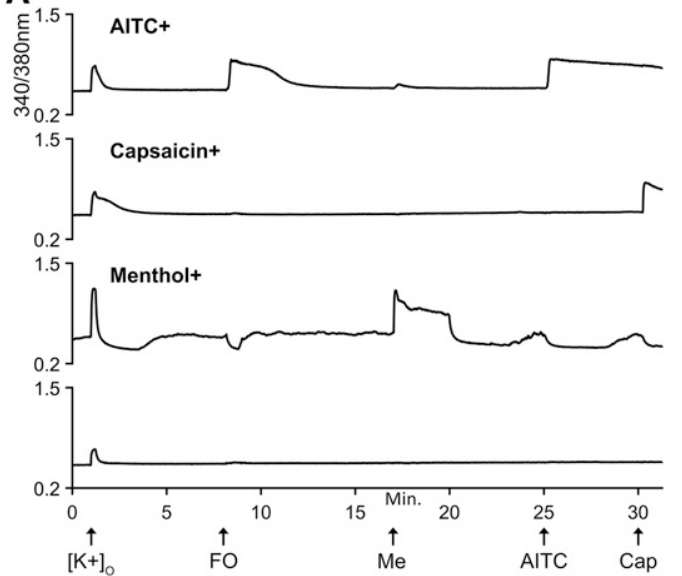

C
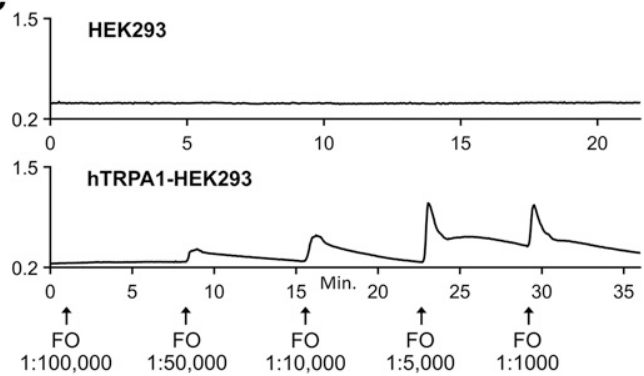

B
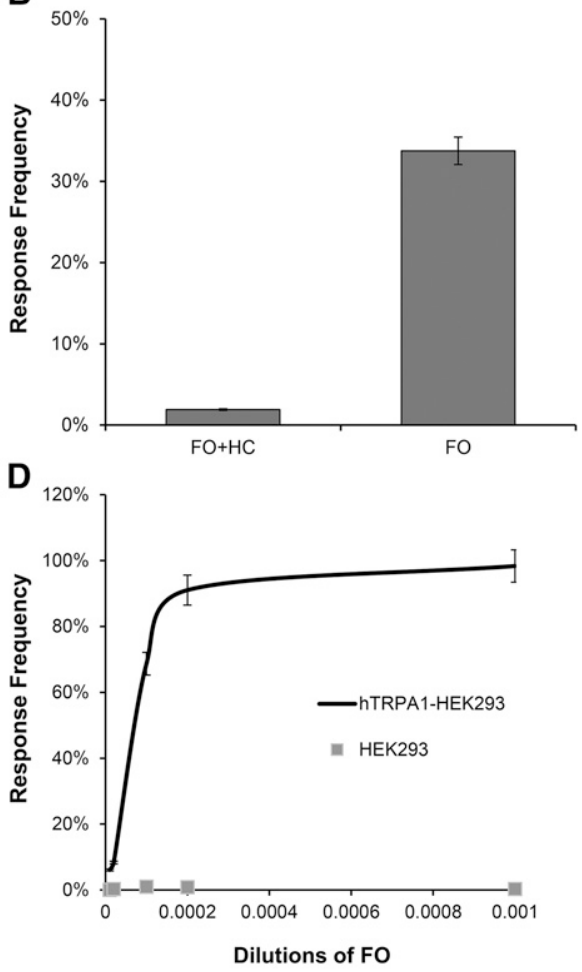

Fig. 1. FO activates mouse and human TRPA1. (A) Representative calcium imaging traces of neuronal phenotypes observed among 1328 neurons analyzed from DRG cultures of three adult wildtype C57BL/6 mice. The $y$-axis is the $340 / 380-\mathrm{nm}$ ratio, a measure of intracellular calcium, and $x$-axis is the duration of the experiment in minutes. Arrows on $x$-axis indicate 15 -second treatments with each stimulus. AITC, $100 \mu \mathrm{M}$ allyl isothiocyanate; Cap, $300 \mathrm{nM}$ capsaicin; FO, 1:10,000-fold diluted fennel oil; $\left[\mathrm{K}^{+}\right]_{\mathrm{o}}$, $30 \mathrm{mM}$ extracellular $\mathrm{K}^{+}$; $\mathrm{Me}, 400 \mu \mathrm{M}$ menthol. (B) Response frequency, measured as percentage of cells activated, of FO (1:10,000-fold diluted) responses in presence and absence of $30 \mu \mathrm{M}$ HC-030031 (HC), a selective TRPA1 antagonist. (C) Representative calcium imaging traces for FO dilutions ( $x$-axis) in normal HEK293 cells (top) and HEK293 cells stably overexpressing human TRPA1 (bottom). (D) Response frequency of FO dilutions in normal $(n=676)$ and human TRPA1-expressing HEK293 cells $(n=1695)$. experiments. Similar experiments with HEK-GCaMP6 cells transiently transfected with control vector were used to correct for nonspecific responses, if any were observed. One-way analysis of variance with Tukey's test was used to evaluate significant differences between treatments. For paw-licking responses to subcutaneous injections, the duration in seconds ( $y$-axis) over a 15-minute period was averaged for each given treatment ( $x$-axis). For these multiple treatment groups, oneway analysis of variance followed by Dunnett's test was used to test significant differences $(P$ value $<0.05)$.

\section{Results}

FO Selectively Activates Mouse and Human TRPA1. To identify the molecular target of FO, we screened the ability of anethole to stimulate somatosensory neurons of mouse DRG using calcium imaging. Different dilutions of FO were tested. A 10,000-fold dilution of FO (containing $332 \pm 6 \mu \mathrm{M}$ anethole) elicited robust calcium influx in a subset of somatosensory neurons (Fig. 1A). To determine the specific neuronal cell subtypes activated by FO, we applied sequentially the TRP channel agonists menthol, AITC, and capsaicin, which activate TRPM8, TRPA1, and TRPV1, respectively, and allow for the segregation of neurons based on TRP channel expression (Teichert et al., 2012a,b, 2014; Memon et al., 2017). In summary, $\sim 38 \%$ neurons responded to $\mathrm{FO}, \sim 27 \%$ to menthol, $\sim 44 \%$ to AITC, and $\sim 47 \%$ to capsaicin. The robust responses (calcium influx) to FO in DRG neurons positively correlated with responses to AITC: at a 1:10,000-fold dilution of FO, $\sim 90 \%$ of FO-sensitive neurons responded to $100 \mu \mathrm{M}$ AITC, and $\sim 80 \%$ of neurons that responded to $100 \mu \mathrm{M}$ AITC also responded to a 1:10,000-fold dilution of FO. Also, 32\% of FOsensitive neurons were both AITC- and capsaicin-sensitive.

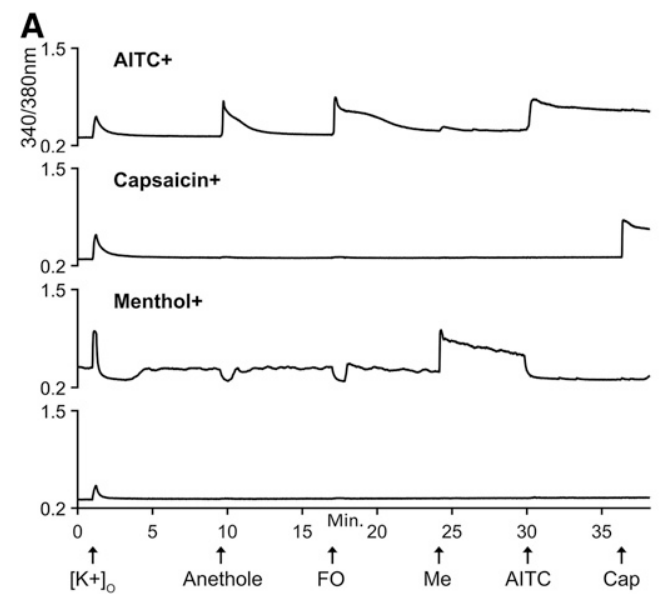

B

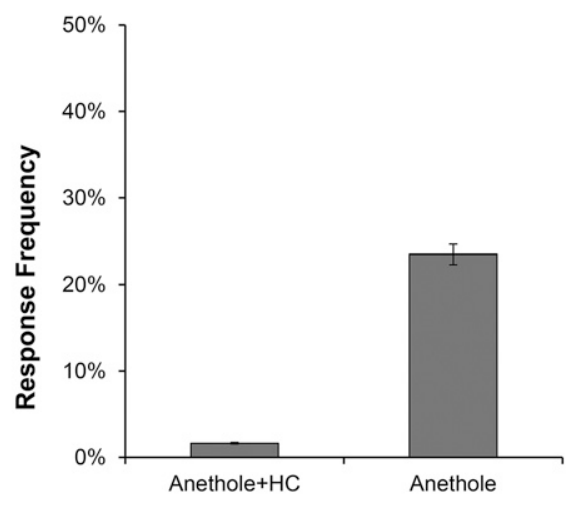

Fig. 2. Anethole elicits calcium response in TRPA1-expressing mouse DRG neurons. (A) Representative calcium imaging traces of neuronal phenotypes observed among 1375 neurons analyzed from DRG cultures of three adult wild-type C57BL/6 mice. The $y$-axis is the $340 / 380$-nm ratio, a measure of intracellular calcium, and $x$-axis is the duration of the experiment in minutes. Arrows on $x$-axis indicate 15 -second treatment with given stimuli. AITC, $100 \mu \mathrm{M}$ allyl isothiocyanate; anethole, $130 \mu \mathrm{M}$ trans-anethole; Cap, $300 \mathrm{nM}$ capsaicin; FO, 1:10,000-fold diluted fennel oil; $\left[\mathrm{K}^{+}\right]_{o}, 30 \mathrm{mM}$ extracellular $\mathrm{K}^{+}$; $\mathrm{Me}, 400 \mu \mathrm{M}$ menthol. (B) Response frequency $(n=964)$ of $130 \mu \mathrm{M}$ anethole in presence and absence of $30 \mu \mathrm{M}$ HC-030031 (HC), a selective TRPA1 antagonist. 
Despite the activation of multiple AITC-sensitive neuronal subtypes, the FO-elicited calcium responses in DRG neurons were completely blocked by cotreatment with $30 \mu \mathrm{M}$ HC-030031, a selective TRPA1 antagonist (Eid et al., 2008), suggesting selective activation of mouse TRPA1 by FO (Fig. 1B).

Similar to mouse DRG neurons, FO robustly and selectively induced calcium influx in HEK293 cells stably overexpressing human TRPA1 (hTRPA1-HEK293) without eliciting any response in normal/wild-type HEK293 cells (Fig. 1, C and D). Similar to results from the neuronal cultures, activation of numerous other TRP channels of interest in TRPoverexpressing HEK293 cells (i.e., TRPM2, M8, V1, V2, V3, or V4) was not observed using a fennel seed extract at concentrations $\leq 2 \mathrm{mg} / \mathrm{ml}$ containing $135 \pm 6 \mu \mathrm{M}$ anethole (Supplemental Fig. 1). Collectively, these results show that FO activates mouse and human TRPA1 with a high degree of selectivity relative to other related TRP and/or calcium channels.

Anethole Is the Major Component of FO. FO extract is composed of a complex mixture of chemicals (Badgujar et al., 2014). To determine specific chemical components that may be responsible for the activation of TRPA1, we first subjected FO to untargeted GC-MS analysis, scanning for analytes between $m / z 40$ and 400. Table 1 shows that FO consists of multiple components; the compound exhibiting the greatest relative peak area $(\sim 78 \%)$ was predicted to be trans-anethole, based on electron impact mass spectral matching ( $97.52 \%$ confidence). The presence of trans-anethole was further confirmed using pure trans-anethole as a standard (Supplemental Fig. 2). Therefore, we hypothesized that trans-anethole may mimic the activity of FO on TRPA1.

Anethole Selectively Activates TRPA1 Channels in Mouse Somatosensory Neurons. Similar to FO, pure anethole also selectively elicited calcium influx in AITCsensitive (i.e., TRPA1-expressing) sensory neurons cultured from mouse DRG. As shown in Fig. 2A, anethole elicited robust calcium influx in AITC-sensitive neurons but not in neurons that were unresponsive to AITC, such as neurons that were responsive to capsaicin only (TRPV1-expressing) or menthol only (TRPM8-expressing). As with FO, anetholeevoked calcium responses were also completely abolished by the TRPA1 antagonist HC-030031, suggesting selective activation of the mouse TRPA1 channel by anethole (Fig. 2B). Of note, the dip in the baseline of TRPM8-expressing neurons during anethole and FO application (Fig. 1A; Fig. 2A) is due to evaporative cooling that occurs following the change in bath solution (Teichert et al., 2014).

As TRP channel agonists mediate chemesthesis via sensory neurons of the TG, we also assessed activation of calcium flux by anethole in TG neurons. Again, anethole selectively induced calcium influx in TRPA1-expressing TG neurons, with

TABLE 1

GC-MS analysis of fennel oil

\begin{tabular}{lccc}
\hline \multicolumn{1}{c}{$\begin{array}{c}\text { Predicted } \\
\text { Chemical }\end{array}$} & $\begin{array}{c}\text { Retention } \\
\text { Time }\end{array}$ & $\begin{array}{c}\text { Percentage of } \\
\text { Total Peak Area }\end{array}$ & $\begin{array}{c}\text { \% Prediction } \\
\text { Accuracy }\end{array}$ \\
\hline$\alpha$-Phellandrene & $2.681-2.702$ & 1.20 & 96.12 \\
D-Limonene & $2.883-2.921$ & 2.22 & 98.16 \\
Fenchone & $3.310-3.326$ & 15.22 & 96.56 \\
Estragole & $3.896-3.913$ & 3.25 & 97.66 \\
trans-Anethole & $4.28-4.306$ & 78.11 & 97.52 \\
\hline
\end{tabular}

detectable responses to anethole in $~ 80 \%$ of AITC-responsive neurons (Supplemental Fig. 3).

Anethole Activates TRPA1-Expressing Neuronal Subtypes. TRPA1 is known to mediate pain sensations (Bautista et al., 2006; McNamara et al., 2007; Viana, 2016), with TRPA1-expressing neurons broadly classified into peptidergic and nonpeptidergic neurons (Braz et al., 2005; Barabas et al., 2012). Therefore, we further evaluated which specific neuronal subtypes of TRPA1-expressing neurons were activated by anethole, compared with AITC. TRPV1-expressing neurons, selectively activated by capsaicin, also mediate pain sensation and were included in this analysis for comparison with TRPA1-expressing neurons. Peptidergic neurons express CGRP and substance P, which are important in the transmission of pain and the development of neurogenic inflammation and edema. Nonpeptidergic neurons bind IB4 and mediate the affective component (unpleasantness) of pain (Braz et al., 2005; Barabas et al., 2012). Using transgenic mice in which GFP expression is driven by the CGRP promoter in CGRP-expressing neurons (Gong et al., 2003), combined with staining neurons with Alexa Fluor 568 IB4 (Barabas et al., 2012), we were able to differentiate peptidergic and nonpeptidergic neuronal subtypes in DRG cell cultures. Based on CGRP expression or IB4 staining, $~ 87 \%$ of both anetholeresponsive (anethole + ) and AITC-responsive (AITC + ) neurons were CGRP + and/or IB4+ compared with $\sim 70 \%$ of capsaicin-responsive (capsaicin + ) neurons (Fig. 3). The majority of anethole + neurons $(65 \% \pm 4 \%)$ were IB4 + , and the remaining were CGRP $+(5 \% \pm 1 \%)$ or colabeled for CGRP + and IB4 $+(17 \% \pm 5 \%)$. Similarly, AITC + neurons were mostly IB $4+(59 \% \pm 4 \%)$, while $7 \% \pm 1 \%$ were CGRP + , and $20 \% \pm$ $4 \%$ were CGRP + and IB4+. In contrast, capsaicin + neurons were mostly colabeled with CGRP + and IB4 $+(36 \% \pm 3 \%)$, while the proportions of CGRP + neurons were $19 \% \pm 5 \%$ and $15 \% \pm 3 \%$ for IB $4+$. These results suggest that anethole, similar to AITC, primarily activates nonpeptidergic (IB4+) and some peptidergic $(\mathrm{CGRP}+)$ neuronal subtypes that express TRPA1.

Anethole Activates Human TRPA1 Channels. Similar to FO, pure anethole also elicited calcium influx in hTRPA1HEK293 cells with an $\mathrm{EC}_{50}$ of $86 \mu \mathrm{M}$, without eliciting any response in normal wild-type HEK293 cells (Fig. 4). The magnitude of calcium response for anethole peaked between 130 and $660 \mu \mathrm{M}$, beyond which desensitization became apparent. Hence, the $\mathrm{EC}_{50}$ for anethole was determined using average response frequency (i.e., the percentage of cells responding to anethole at a given concentration) (Fig. 4B), which was unaffected during the time period of the experiment. Furthermore, pure anethole induced whole-cell transmembrane currents in hTRPA1-HEK293 cells (Fig. 5A). For these experiments, the extracellular solution for whole-cell patch clamp contained tetraethylammonium to suppress the influence of endogenous $\mathrm{K}^{+}$conductance (Jiang et al., 2002; Yarishkin et al., 2008; Ponce et al., 2018) to TRPA1-mediated responses in these cells. Results in Fig. 5B show the I-V relationship of transmembrane current measured before and after anethole treatment. Based on the dose-response relationship shown in Fig. 5C, anethole induced whole-cell currents in hTRPA1-HEK293 cells with an $\mathrm{EC}_{50}$ of $80.8 \mu \mathrm{M}$, essentially the same $\mathrm{EC}_{50}$ as observed for pure anetholeinduced calcium flux in these cells. Of note, the dose-response relationship was determined with whole-cell patch clamp 


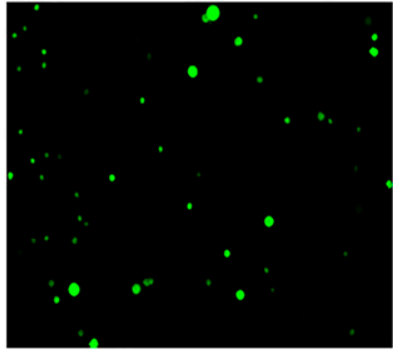

CGRP+

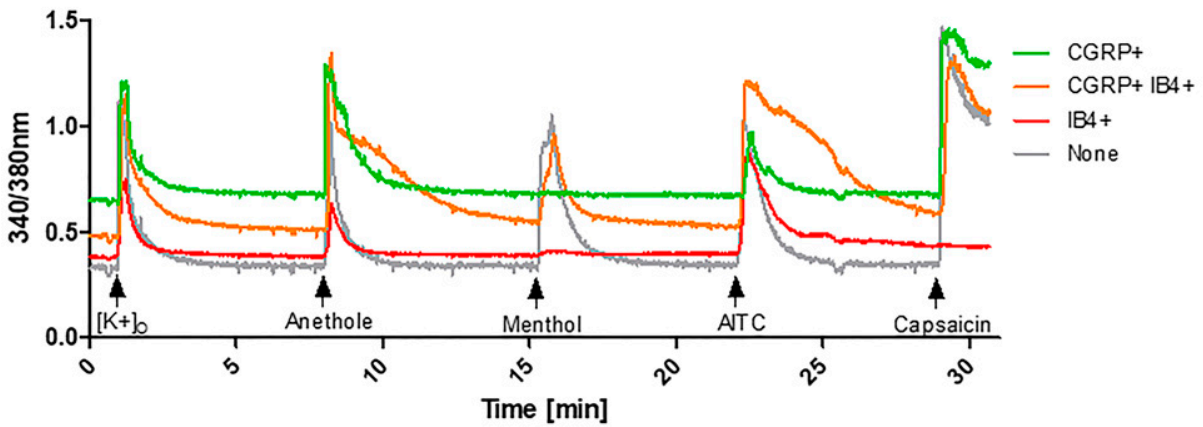

Anethole+
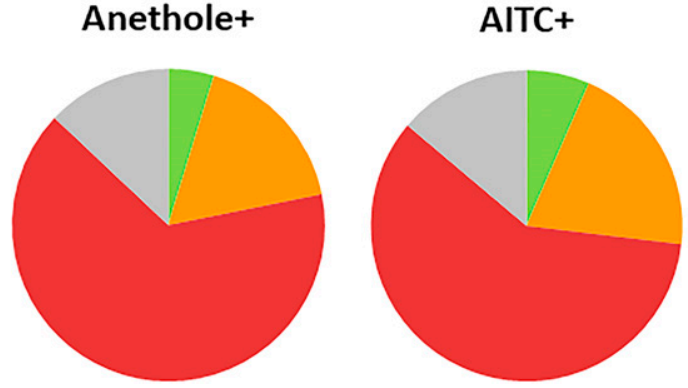

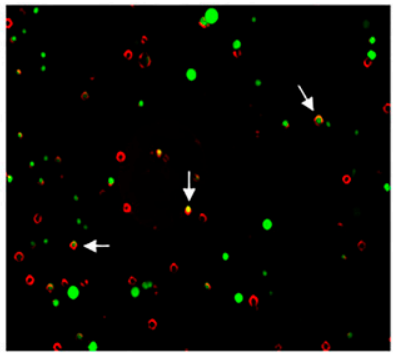

CGRP+ IB4+

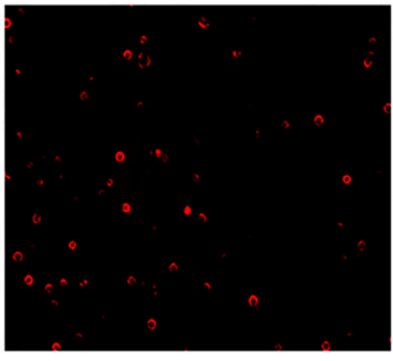

IB4+

Fig. 3. Distribution of peptidergic and nonpeptidergic neuronal subtype neurons activated by anethole, AITC, and capsaicin. (Top) Representative images show CGRP+, CGRP+ IB4+ (overlaid images of CGRP+ and IB4+), and IB4+ neurons (from left to right). White arrows point at examples of CGRP+ and IB4+ neurons. (Middle) Representative traces for each neuronal subtype. (Bottom) Pie charts show average distribution of neuronal cell types, i.e., CGRP+ (green), CGRP+ IB4+ (yellow), IB4+ (red), and CGRP- IB4- or none (gray) activated by anethole $(n=$ 504), AITC $(n=731)$ and capsaicin $(n=$ 945) from DRG cultures of three adult CD-1 mice.

(Fig. 5C) using different cells treated with different concentrations of anethole, as opposed to serial application, to avoid the potential influence of desensitization caused by anethole. However, based on the similarity of the $\mathrm{EC}_{50}$ determined from these two experiments, it appears that desensitization did not drastically affect the $\mathrm{EC}_{50}$ of anethole.

Anethole Is a Nonelectrophilic Agonist of TRPA1. Reactive molecules and electrophiles such as AITC activate TRPA1 by covalent modification of key cysteine and lysine residues located on the intracellular $\mathrm{N}$-terminal portion of the channel (Macpherson et al., 2007). However, nonelectrophilic
TRPA1 agonists such as menthol and propofol interact with Ser-873 and Thr-874 residues located in transmembrane five of TRPA1 (Xiao et al., 2008). To differentiate the mechanism of TRPA1 activation by anethole via binding the known electrophile versus nonelectrophile agonist sites, we compared TRPA1 activation of wild-type TRPA1 and the "ST" mutant (S873V+T874L) form of TRPA1 by AITC and anethole with and without preincubation of the agonist treatment solutions with GSH, as previously described (Shapiro et al., 2013). Human TRPA1 activation by AITC was essentially abolished when the solution of AITC was preincubated with
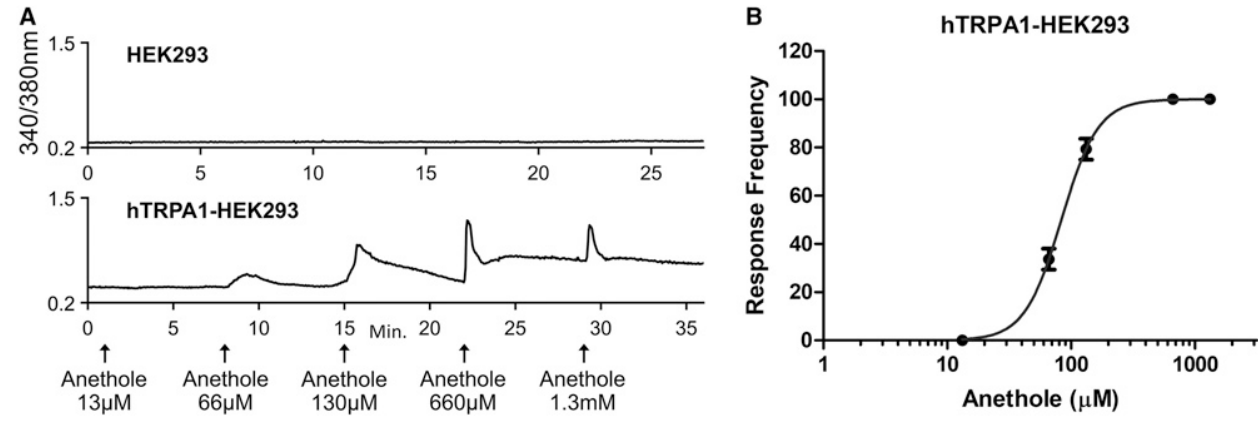

Fig. 4. Anethole elicits calcium response in hTRPA1-HEK293 cells. (A) Representative calcium imaging traces for anethole dose response over time ( $x$-axis) in normal HEK293 cells (top) and HEK293 cells stably overexpressing human TRPA1 (bottom). The $y$-axis is the $340 / 380-\mathrm{nm}$ ratio, a measure of intracellular calcium, and $x$-axis is the duration of the experiment in minutes. Arrows on $x$-axis indicate 15 -second treatment with given stimuli. (B) Response frequency, measured as percentage of cells activated, of anethole dose response in normal human TRPA1expressing HEK293 cells $(n=1195)$. 

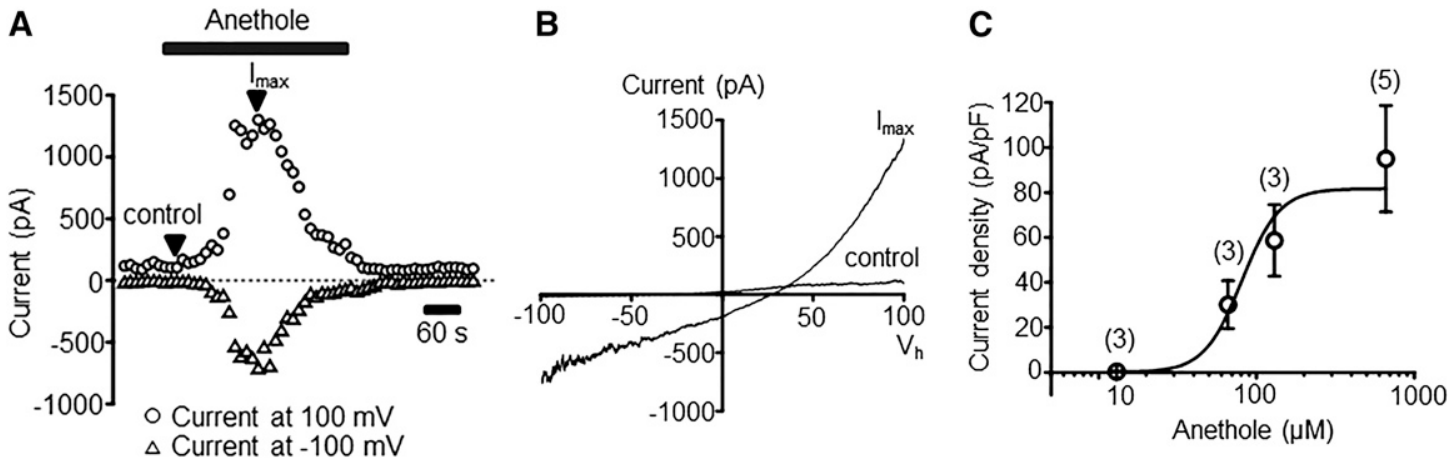

Fig. 5. Anethole induces transmembrane currents in hTRPA1-HEK293 cells. (A) A representative time course of current measured at the indicated holding potentials $\left(\mathrm{V}_{\mathrm{h}}\right)$ from HEK293 cells stably overexpressing human TRPA1. Anethole was applied at a concentration of $66 \mu \mathrm{M}$. (B) I-V relationship of transmembrane current measured before (control) and during administration of anethole (maximum current, $\mathrm{I}_{\max }$ ). (C) Dose-response relationship. Circles represent mean values of $\mathrm{I}_{\max }$ recorded at $\mathrm{V}_{\mathrm{h}}=100 \mathrm{mV}$. Error bars indicate S.E.M. The solid line is the best fit with logistic function. $\mathrm{EC}_{50}=80.8$ $\mu \mathrm{M}$. Shown in parentheses are the number of cells.

$20 \mathrm{mM}$ GSH, a nucleophile that forms reversible covalent bonds with AITC, effectively reducing the concentration of free AITC in the treatment solutions by rendering it nonreactive in the conjugated form (Fig. 6). Conversely, when anethole was pretreated with $20 \mathrm{mM}$ GSH for 10 minutes, no inhibition of TRPA1 activation was observed (Fig. 6). Also shown in Fig. 6, HEK293 cells transiently transfected with the hTRPA1$\mathrm{S} 873 \mathrm{~V}+\mathrm{T} 874 \mathrm{~L}$ mutant showed a slight decrease in response to AITC but a near complete loss of response to anethole compared with cells transfected with wild-type hTRPA1. Further, GSH did not reduce activation of the hTRPA1-ST mutant by anethole, as observed for AITC. These results imply that anethole is a nonelectrophilic agonist that binds to the ST binding site on TRPA1.

Desensitization of TRPA1-Mediated Anethole Responses. TRPA1 agonists such as AITC, limonene, and eugenol often show bimodal activity on TRPA1 channels such that repetitive applications desensitize the channels (Chung et al., 2014; Kaimoto et al., 2016; Kistner et al., 2016). Similarly, the dose-response experiment shown in Fig. 4 hinted at the possibility of desensitization of anetholeelicited calcium responses in hTRPA1-HEK293 cells once the response amplitude reached a maximum (between 130 and $660 \mu \mathrm{M})$. Hence, anethole was tested for the bimodal activation of TRPA1. Anethole was repetitively applied to mouse DRG neurons and hTRPA1-HEK293 cells for a total of three times over a period of 15 minutes (Fig. 7). The magnitude of each anethole-elicited calcium response was compared with that of the first anethole application for each cell type. As shown in Fig. 7, significant decreases in the average magnitude of anethole-elicited calcium responses in both mouse DRG neurons (Fig. 7, A and B) and hTRPA1HEK293 cells (Fig. 7, C and D) were observed over time with each sequential treatment with anethole. These results were also consistent with the desensitization observed in whole-cell current recordings in TRPA1-expressing HEK293 cells during anethole treatment (Fig. 5A; Fig. 8). Cumulatively, our results suggest desensitization of TRPA1 mediated anethole responses when stimulated repetitively.

Anethole Does Not Induce Nocifensive Responses in Mice. Many known TRPA1 agonists are perceived as pungent and noxious, frequently eliciting nocifensive behaviors such as paw lifting and licking when injected subcutaneously in mice (Kwan et al., 2006; Everaerts et al., 2011). However, when injected subcutaneously in the mouse hind paw, neither $20 \mu \mathrm{l}$ of a $6.6 \mathrm{mM}$ anethole solution nor $20 \mu \mathrm{l}$ of a $66 \mathrm{mM}$ anethole solution induced nocifensive (i.e., increased paw-licking duration) responses (Fig. 9). In contrast, injection of $20 \mu \mathrm{l}$ of a $10 \mathrm{mM}$ AITC solution induced significant paw-licking (Fig. 9) and paw-lifting behaviors. As neither vehicle- nor anetholetreated mice displayed paw lifting, only paw-licking duration was compared for the given treatments shown in Fig. 9. These data imply that anethole is substantially less or perhaps even nonirritating relative to AITC, although the mechanisms of this effect require further study.

\section{Discussion}

This study identified anethole in FO as a selective agonist of mouse and human TRPA1. FO and its major component, trans-anethole, selectively activated TRPA1 expressed in

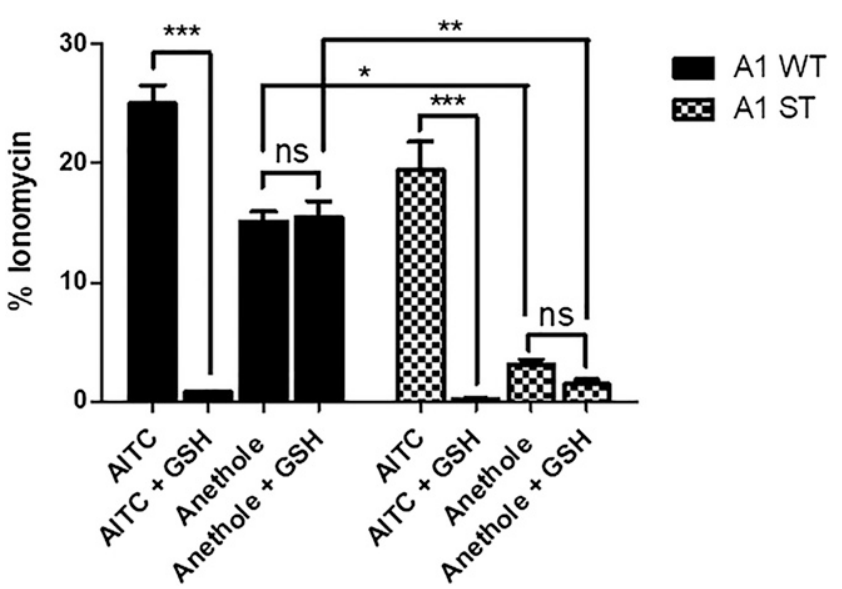

Fig. 6. Anethole is a nonelectrophilic agonist of TRPA1. HEK293GCaMP6-overexpressing cells transiently transfected with wild-type hTRPA1 (A1 WT) and hTRPA1-ST (A1 ST) mutant plasmid robustly respond to AITC and anethole. Pretreatment of AITC with $20 \mathrm{mM}$ GSH for 10 minutes abolished AITC responses in both A1 WT and A1 ST cells $(* * * P<0.001)$, while the response to anethole when pretreated with GSH was not affected. In cells transfected with (A1 ST) plasmid, the response to AITC was not significantly affected, while response to anethole was significantly diminished $(* P<0.05 ; * * P<0.01)$. Data are the average response \pm S.E.M. of treatments $(x$-axis) normalized to the ionomycin response $(y$-axis) for the respective plasmid $(n=3)$. ns, not significant. 

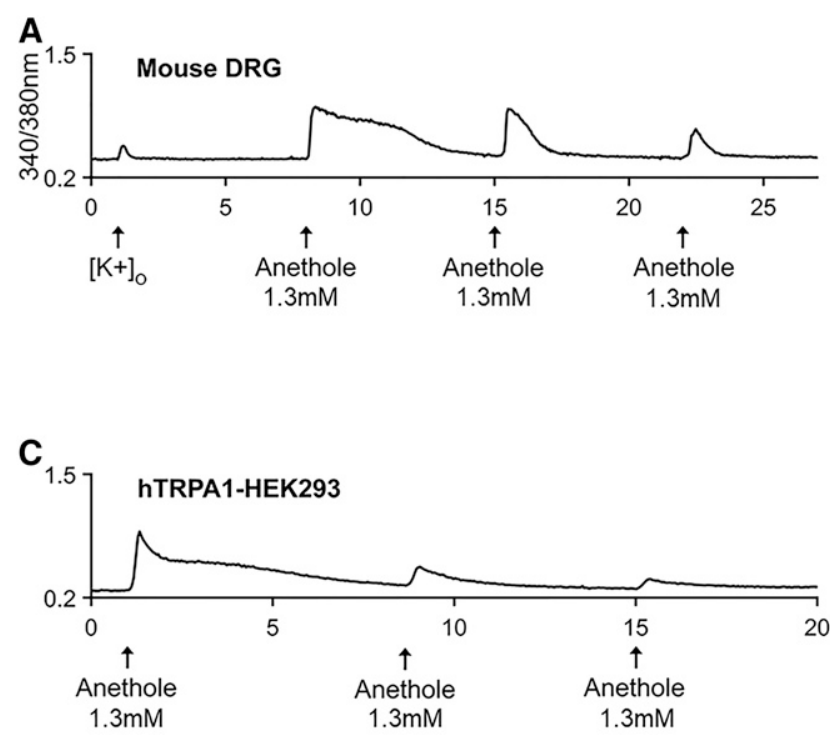

B
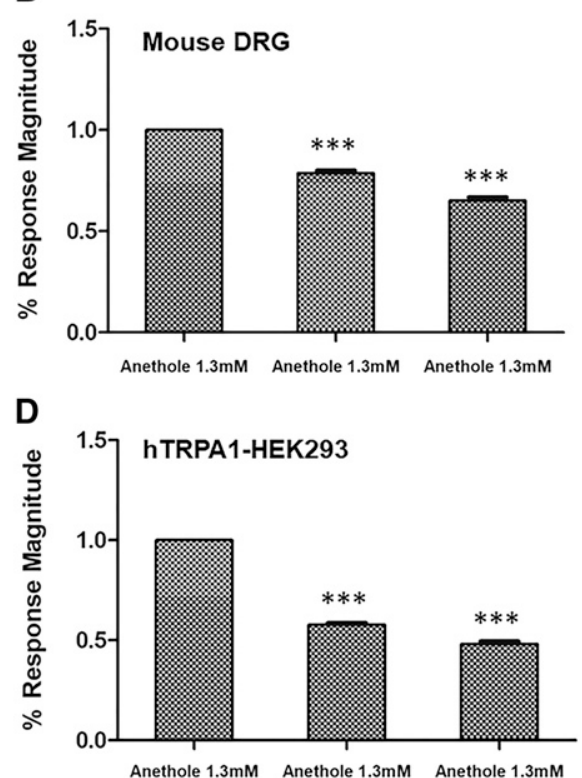

Fig. 7. Desensitization of anethole-elicited calcium responses. Representative calcium imaging trace illustrating desensitization of calcium responses, indicated by the declining magnitude of responses upon the second and third applications of anethole, each elicited by a 15 -second application of $1.3 \mathrm{mM}$ anethole on mouse DRG neurons (A) and HEK293 cells overexpressing human TRPA1 (C). (B and D) The average percentage response magnitude of second and third responses in mouse DRG neurons $(n=372)$ and hTRPA1-HEK293 cells $(n=833)$ as a percentage of the first response to $1.3 \mathrm{mM}$ anethole, indicating significant desensitization of anethole-induced calcium responses over time $(* * * P<0.001)$. The second and third responses to anethole $(x$-axis $)$ were normalized to the first response of each neuron or HEK293 cell. Average percentage response \pm S.E.M. values are presented.

mouse DRG and TG neurons, as well as human TRPA1 expressed in HEK293 cells, determined using both calcium imaging and electrophysiology. In all cases, the responses to FO or pure anethole could be blocked completely by a selective antagonist of TRPA1. FO also did not activate other human TRP channels expressed in HEK293 cells. It was further found that TRPA1 activation by anethole was due to binding to the menthol/propofol ST binding site on TRPA1, and repeated application of anethole led to desensitization of TRPA1mediated anethole response. Finally, anethole did not elicit nocifensive responses typical of many TRPA1 agonists, notably AITC, which was used here as a positive control. To the best of our knowledge, this is the first study to identify TRPA1 as a primary molecular target of $\mathrm{FO}$ and its major component anethole.

Traditionally, TRPA1, similar to TRPV1, is considered to be a nociceptive ion channel (McNamara et al., 2007; Kremeyer et al., 2010; Bautista et al., 2013). As such, antagonists of TRPA1 have been in preclinical and clinical development for pain relief (Eid et al., 2008; Zhao et al., 2012; Gui et al., 2014). Interestingly, treatment with anethole has been reported to be analgesic and anti-inflammatory in mouse models of pain (Ritter et al., 2013), with similar observations made with other "pleasant" TRPA1 channel agonists, such as limonene and eugenol (Leamy et al., 2011; Wang et al., 2013; Chung et al., 2014; Zhai et al., 2014; Bressan et al., 2016; Kaimoto et al., 2016). These TRPA1 agonists are believed to assert analgesic activity by activating and subsequently desensitizing the TRPA1 channel (Story et al., 2003; Akopian et al., 2007; Leamy et al., 2011; Zhai et al., 2014; Bressan et al., 2016; Kaimoto et al., 2016). Here, we observed significant differences between the ability of AITC and anethole to elicit nocifensive behavioral responses (i.e., paw licking). Specifically, anethole appeared to be less or nonirritating (Fig. 9).
A

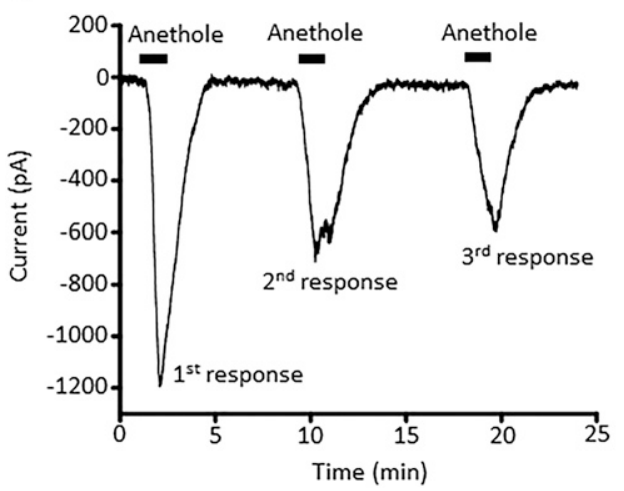

B

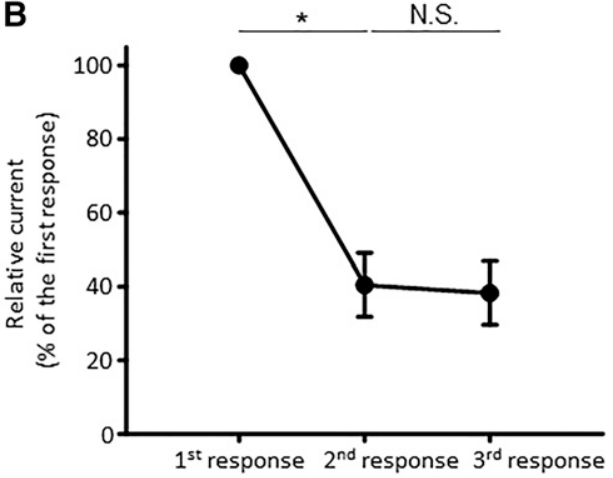

Fig. 8. Repetitive stimulation of TRPA1 by anethole reveals a desensitization of the TRPA1-mediated whole-cell current to the agonist. (A) A representative trace of the whole-cell current recorded from an hTRPA1-HEK293 cell with the plasma membrane potential clamped at $-70 \mathrm{mV}$. Application of anethole $(100 \mu \mathrm{M})$ is indicated by bars above the trace. (B) Statistical analysis of results illustrated in (A). Magnitude of the second and third responses was normalized to that of the first response. Shown are the mean \pm S.E. M. values. ${ }^{*} P<0.05 ;$ N.S., not significant $(P>0.05)$; paired-sample $t$ test, $n=4$ cells. Anethole was applied for 1.5 minutes. 

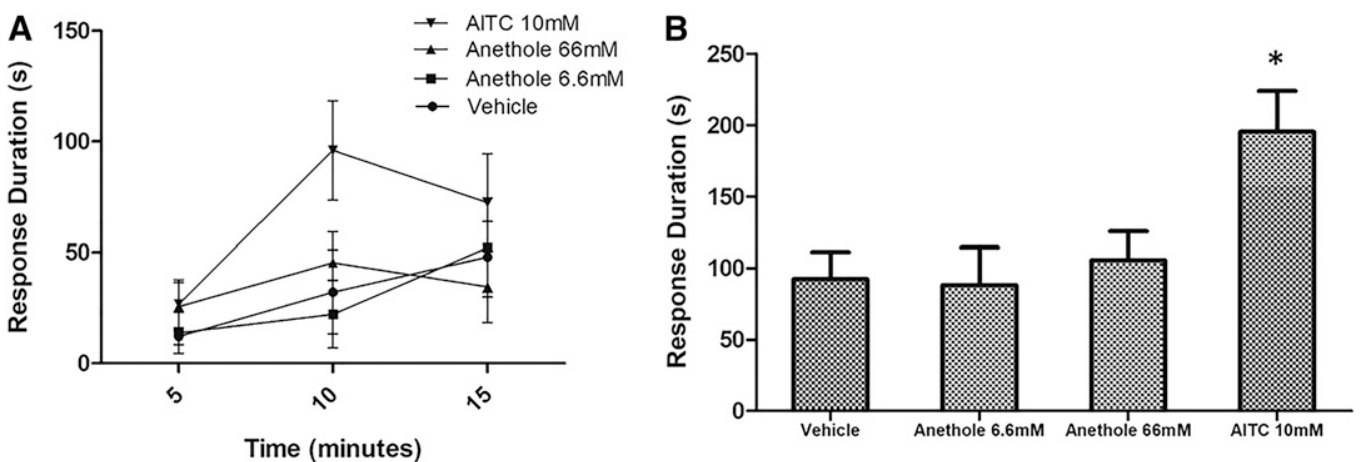

Fig. 9. Nocifensive response induced by subcutaneous injection of anethole. Paw-licking behavior of adult wild-type C57BL/6 mice was recorded as response duration in seconds \pm S.E.M. ( $y$-axis) observed over 15 -minute duration after subcutaneous injection of given treatments in left hind paw. (A) Response duration of paw-licking behavior every 5 minutes. (B) Sum of paw-licking response duration for given treatments ( $x$-axis) during 15 -minute observation period. DRG Obs. was used as vehicle. Significant difference $(* P<0.05)$ in paw-licking response duration was observed between vehicletreated $(n=6)$ and $10 \mathrm{mM}$ AITC- treated $(n=7)$ mice, while the difference between vehicle- and $6.6 \mathrm{mM}$ anethole- treated $(n=6)$ or $66 \mathrm{mM}$ anetholetreated $(n=5)$ mice was not significant.

Consistent with this observation and previous findings for related TRPA1 agonists, repeated exposure to anethole desensitized TRPA1-mediated anethole responses in mouse DRG neurons (Fig. 7, A and B) and hTRPA1-HEK293 cells (Fig. 7, C and D; Fig. 8). Similar effects have been reported for TRPV1 agonists, such as capsaicin, which also mediate analgesia through tachyphylaxis (desensitization) of the TRPV1 channel, changes in TRPV1 expression, and/or neuronal toxicity (Jo et al., 2017), mechanisms by which capsaicinbased topical creams mediate pain relief (Patapoutian et al., 2009). Thus, it is possible that anethole, similar to other TRPA1 (and TRPV1) agonists, exerts its pharmacological effects, at least in part, by activating and then desensitizing the TRPA1 channel.

However, AITC also desensitizes TRPA1 (Akopian et al., 2007; Kistner et al., 2016) but still elicits nocifensive behaviors. Therefore, it is also possible that slight differences in the specific subtypes of TRPA1-expressing neurons that are activated by anethole and AITC (Fig. 3) could contribute to the differences in behavioral responses elicited by AITC and anethole. Another likely possibility involves differences in the chemical mechanism of TRPA1 activation, which is ultimately rooted in the inherent differences in the chemical reactivity of anethole, AITC, and other TRPA1 agonists for which differences in pain-producing potential have been reported (Chung et al., 2014; Kaimoto et al., 2016). As alluded earlier, many agonists of TRPA1 are perceived as pungent compounds, which activate TRPA1 through covalent modification. However, some more pleasant compounds such as menthol (Xiao et al., 2008), limonene (Kaimoto et al., 2016), and eugenol (Chung et al., 2014), which are nonreactive in the absence of metabolic bioactivation (e.g., the quinone methide intermediate reported for eugenol is reactive), also activate TRPA1 independent of covalent modification and do not show the typical pain-producing and irritating effects. Therefore, the sweet and licorice-like FO and anethole described in this study likely fall into this interesting category of pleasant TRPA1 agonists that are nonreactive and apparently less or nonirritating agonists. It is presently unclear whether the differences in activation mechanisms ultimately account for the divergence in physiologic effects elicited by different TRPA1 channel agonists, but the evidence suggests this may be an important factor. As such, it is also possible that the reactive nature of many pungent and painful TRPA1 agonists may lead to localized tissue injury and inflammatory hyperalgesia independent of TRPA1, and that this effect may broadly sensitize neurons and increase pain perception. However, these ideas will require further study.

To summarize, our results show that TRPA1 is a prominent target of FO and anethole in sensory neurons of DRG and TG. Anethole is similar to AITC in terms of activating TRPA1 and desensitizing the channel, but unlike AITC, anethole does not appear to covalently modify TRPA1 and fails to induce a detectable change in prototypical nocifensive behaviors in mice. These differences highlight the disparate nature of TRPA1 agonists and the need to further explore how different TRPA1 agonists affect neuronal functions, inflammatory responses, and pain perception.

\section{Acknowledgments}

We thank Dr. David Ginty (Harvard Medical School) for providing transgenic CGRP-GFP mice used in this study. We thank Kevin Chase for developing $\mathrm{R}$ functions used to analyze calcium imaging data and My Huynh for her assistance in preparing figures.

\section{Authorship Contributions}

Participated in research design: Memon, Yarishkin, Reilly, Križaj, Olivera, Teichert.

Conducted experiments: Memon, Yarishkin, Teichert.

Performed data analysis: Memon, Yarishkin, Teichert.

Wrote or contributed to the writing of the manuscript: Memon, Yarishkin, Reilly, Križaj, Teichert.

\section{References}

Akopian AN, Ruparel NB, Jeske NA, and Hargreaves KM (2007) Transient receptor potential TRPA1 channel desensitization in sensory neurons is agonist dependent and regulated by TRPV1-directed internalization. J Physiol 583:175-193.

Badgujar SB, Patel VV, and Bandivdekar AH (2014) Foeniculum vulgare Mill: a review of its botany, phytochemistry, pharmacology, contemporary application, and toxicology. BioMed Res Int 2014:842674

Barabas ME, Kossyreva EA, and Stucky CL (2012) TRPA1 is functionally expressed primarily by IB4-binding, non-peptidergic mouse and rat sensory neurons. PLoS One 7:e47988.

Bautista DM, Jordt S-E, Nikai T, Tsuruda PR, Read AJ, Poblete J, Yamoah EN, Basbaum AI, and Julius D (2006) TRPA1 mediates the inflammatory actions of environmental irritants and proalgesic agents. Cell 124:1269-1282.

Bautista DM, Pellegrino M, and Tsunozaki M (2013) TRPA1: a gatekeeper for inflammation. Annu Rev Physiol 75:181-200.

Bautista DM, Siemens J, Glazer JM, Tsuruda PR, Basbaum AI, Stucky CL, Jordt S$\mathrm{E}$, and Julius D (2007) The menthol receptor TRPM8 is the principal detector of environmental cold. Nature 448:204-208.

Braz JM, Nassar MA, Wood JN, and Basbaum AI (2005) Parallel "pain" pathways arise from subpopulations of primary afferent nociceptor. Neuron 47:787-793. 
Bressan E, Touska F, Vetter I, Kistner K, Kichko TI, Teixeira B, Picolo G, Cury Y, Lewis RJ, and Fischer MJM (2016) Crotalphine desensitizes TRPA1 ion channels to alleviate inflammatory hyperalgesia. Pain 157:2504-2516.

Caterina MJ, Schumacher MA, Tominaga M, Rosen TA, Levine JD, and Julius D (1997) The capsaicin receptor: a heat-activated ion channel in the pain pathway. Nature 389:816-824.

Chung G, Im ST, Kim YH, Jung SJ, Rhyu M-R, and Oh SB (2014) Activation of transient receptor potential ankyrin 1 by eugenol. Neuroscience 261:153-160.

Deering-Rice CE, Johansen ME, Roberts JK, Thomas KC, Romero EG, Lee J, Yost GS, Veranth JM, and Reilly CA (2012) Transient Receptor Potential Vanilloid-1 (TRPV1) Is a Mediator of Lung Toxicity for Coal Fly Ash Particulate Material. Mol Pharmacol 81:411-419.

Deering-Rice CE, Romero EG, Shapiro D, Hughen RW, Light AR, Yost GS, Veranth JM, and Reilly CA (2011) Electrophilic components of diesel exhaust particles (DEP) activate transient receptor potential ankyrin-1 (TRPA1): a probable mechanism of acute pulmonary toxicity for DEP. Chem Res Toxicol 24:950-959.

Deering-Rice CE, Shapiro D, Romero EG, Stockmann C, Bevans TS, Phan QM, Stone BL, Fassl B, Nkoy F, Uchida DA, et al. (2015) Activation of transient receptor potential ankyrin-1 by insoluble particulate material and association with asthma Am J Respir Cell Mol Biol 53:893-901.

Eid SR, Crown ED, Moore EL, Liang HA, Choong KC, Dima S, Henze DA, Kane SA, and Urban MO (2008) HC-030031, a TRPA1 selective antagonist, attenuates inflammatory- and neuropathy-induced mechanical hypersensitivity. Mol Pain 4:48.

Everaerts W, Gees M, Alpizar YA, Farre R, Leten C, Apetrei A, Dewachter I, van Leuven F, Vennekens R, De Ridder D, et al. (2011) The capsaicin receptor TRPV1 is a crucial mediator of the noxious effects of mustard oil. Curr Biol 21:316-321.

Friedland K and Harteneck C (2017) Spices and odorants as TRP channel activators, in Springer Handbook of Odor (Buettner A ed) pp 85-86, Springer International Publishing, Cham, Switzerland.

Gong S, Zheng C, Doughty ML, Losos K, Didkovsky N, Schambra UB, Nowak NJ, Joyner A, Leblanc G, Hatten ME, et al. (2003) A gene expression atlas of the central nervous system based on bacterial artificial chromosomes. Nature 425 917-925.

Gui J, Liu B, Cao G, Lipchik AM, Perez M, Dekan Z, Mobli M, Daly NL, Alewood PF, Parker LL, et al. (2014) A tarantula-venom peptide antagonizes the TRPA1 nociceptor ion channel by binding to the S1-S4 gating domain. Curr Biol 24:473-483.

Jiang B, Sun X, Cao K, and Wang R (2002) Endogenous Kv channels in human embryonic kidney (HEK-293) cells. Mol Cell Biochem 238:69-79.

Jo AO, Lakk M, Frye AM, Phuong TTT, Redmon SN, Roberts R, Berkowitz BA Yarishkin O, and Križaj D (2016) Differential volume regulation and calcium signaling in two ciliary body cell types is subserved by TRPV4 channels. Proc Natl Acad Sci USA 113:3885-3890.

Jo AO, Noel JM, Lakk M, Yarishkin O, Ryskamp DA, Shibasaki K, McCall MA and Križaj D (2017) Mouse retinal ganglion cell signalling is dynamically modulated through parallel anterograde activation of cannabinoid and vanilloid pathways. J Physiol 595:6499-6516.

Jordt S-E, Bautista DM, Chuang H-H, McKemy DD, Zygmunt PM, Högestätt ED, Meng ID, and Julius D (2004) Mustard oils and cannabinoids excite sensory nerve fibres through the TRP channel ANKTM1. Nature 427:260-265.

Kaimoto T, Hatakeyama Y, Takahashi K, Imagawa T, Tominaga M, and Ohta T (2016) Involvement of transient receptor potential A1 channel in algesic and analgesic actions of the organic compound limonene. Eur J Pain 20:1155-1165.

Kistner K, Siklosi N, Babes A, Khalil M, Selescu T, Zimmermann K, Wirtz S, Becker C, Neurath MF, Reeh PW, et al. (2016) Systemic desensitization through TRPA1 channels by capsazepine and mustard oil - a novel strategy against inflammation and pain. Sci Rep 6:28621.

Kremeyer B, Lopera F, Cox JJ, Momin A, Rugiero F, Marsh S, Woods CG, Jones NG Paterson KJ, Fricker FR, et al. (2010) A gain-of-function mutation in TRPA1 causes familial episodic pain syndrome. Neuron 66:671-680.

Kwan KY, Allchorne AJ, Vollrath MA, Christensen AP, Zhang D-SS, Woolf CJ, and Corey DP (2006) TRPA1 contributes to cold, mechanical, and chemical nociception but is not essential for hair-cell transduction. Neuron 50:277-289.

Lamb JG, Romero EG, Lu Z, Marcus SK, Peterson HC, Veranth JM, Deering-Rice $\mathrm{CE}$, and Reilly CA (2017) Activation of human transient receptor potential melastatin-8 (TRPM8) by calcium-rich particulate materials and effects on human lung cells. Mol Pharmacol 92:653-664.
Leamy AW, Shukla P, McAlexander MA, Carr MJ, and Ghatta S (2011) Curcumin ((E,E)-1,7-bis(4-hydroxy-3-methoxyphenyl)-1,6-heptadiene-3,5-dione) activates and desensitizes the nociceptor ion channel TRPA1. Neurosci Lett 503:157-162.

Macpherson LJ, Dubin AE, Evans MJ, Marr F, Schultz PG, Cravatt BF, and Patapoutian A (2007) Noxious compounds activate TRPA1 ion channels through covalent modification of cysteines. Nature 445:541-545.

McNamara CR, Mandel-Brehm J, Bautista DM, Siemens J, Deranian KL, Zhao M, Hayward NJ, Chong JA, Julius D, Moran MM, et al. (2007) TRPA1 mediates formalin-induced pain. Proc Natl Acad Sci USA 104:13525-13530.

Memon T, Chase K, Leavitt LS, Olivera BM, and Teichert RW (2017) TRPA1 expression levels and excitability brake by $\mathrm{K}_{\mathrm{V}}$ channels influence cold sensitivity of TRPA1-expressing neurons. Neuroscience 353:76-86.

Patapoutian A, Tate S, and Woolf CJ (2009) Transient receptor potential channels: targeting pain at the source. Nat Rev Drug Discov 8:55-68.

Phuong TTT, Redmon SN, Yarishkin O, Winter JM, Li DY, and Križaj D (2017) Calcium influx through TRPV4 channels modulates the adherens contacts between retinal microvascular endothelial cells. J Physiol 595:6869-6885.

Ponce A, Castillo A, Hinojosa L, Martinez-Rendon J, and Cereijido M (2018) The expression of endogenous voltage-gated potassium channels in HEK293 cells is affected by culture conditions. Physiol Rep 6:e13663.

Ritter AMV, Domiciano TP, Verri WA Jr, Zarpelon AC, da Silva LG, Barbosa CP, Natali MRM, Cuman RKN, and Bersani-Amado CA (2013) Antihypernociceptive activity of anethole in experimental inflammatory pain. Inflammopharmacology 21:187-197.

Roper SD (2014) TRPs in taste and chemesthesis, in Mammalian Transient Receptor Potential (TRP) Cation Channels (Nilius B and Flockerzi V eds) vol. II, pp 827-871, Springer International Publishing, Cham, Switzerland.

Shapiro D, Deering-Rice CE, Romero EG, Hughen RW, Light AR, Veranth JM, and Reilly CA (2013) Activation of transient receptor potential ankyrin-1 (TRPA1) in lung cells by wood smoke particulate material. Chem Res Toxicol 26:750-758.

Story GM, Peier AM, Reeve AJ, Eid SR, Mosbacher J, Hricik TR, Earley TJ, Hergarden AC, Andersson DA, Hwang SW, et al. (2003) ANKTM1, a TRP-like channel expressed in nociceptive neurons, is activated by cold temperatures. Cell 112: 819-829.

Teichert RW, Memon T, Aman JW, and Olivera BM (2014) Using constellation pharmacology to define comprehensively a somatosensory neuronal subclass. Proc Natl Acad Sci USA 111:2319-2324.

Teichert RW, Raghuraman S, Memon T, Cox JL, Foulkes T, Rivier JE, and Olivera BM (2012a) Characterization of two neuronal subclasses through constellation pharmacology. Proc Natl Acad Sci USA 109:12758-12763.

Teichert RW, Smith NJ, Raghuraman S, Yoshikami D, Light AR, and Olivera BM (2012b) Functional profiling of neurons through cellular neuropharmacology. Proc Natl Acad Sci USA 109:1388-1395.

Viana F (2016) TRPA1 channels: molecular sentinels of cellular stress and tissue damage. J Physiol 594:4151-4169.

Wang S, Dai Y, Kogure Y, Yamamoto S, Zhang W, and Noguchi K (2013) Etodolac activates and desensitizes transient receptor potential ankyrin 1. J Neurosci Res 91:1591-1598

Xiao B, Dubin AE, Bursulaya B, Viswanath V, Jegla TJ, and Patapoutian A (2008) Identification of transmembrane domain 5 as a critical molecular determinant of menthol sensitivity in mammalian TRPA1 channels. J Neurosci 28:9640-9651.

Yarishkin OV, Ryu HW, Park J-Y, Yang MS, Hong S-G, and Park KH (2008) Sulfonate chalcone as new class voltage-dependent $\mathrm{K}+$ channel blocker. Bioorg Med Chem Lett 18:137-140.

Zhai C, Liu Q, Zhang Y, Wang S, Zhang Y, Li S, and Qiao Y (2014) Identification of natural compound carnosol as a novel TRPA1 receptor agonist. Molecules 19: 18733-18746.

Zhao M, Isami K, Nakamura S, Shirakawa H, Nakagawa T, and Kaneko S (2012) Acute cold hypersensitivity characteristically induced by oxaliplatin is caused by the enhanced responsiveness of TRPA1 in mice. Mol Pain 8:55.

Address correspondence to: Tosifa Memon, Department of Pharmacology and Toxicology, University of Utah, 30 S 2000 E, Room 201 Skaggs Hall, Salt Lake City, UT 84112. E-mail: tosifa.memon@utah.edu 